\title{
Frontiera reale e frontiera metaforica nelle opere degli emigrati italiani in Svizzera
}

\author{
Jean-Jacques Marchand \\ Università di Losanna
}

\begin{abstract}
Nelle oltre 250 opere letterarie scritte da emigrati italiani in Svizzera è quasi sempre presente il concetto di frontiera. Ma con il passare del tempo e a seconda dei generi, la frontiera, da discrimine fra un prima e un dopo, da luogo di un trauma spesso rimosso, si trasforma in metafora di un percorso di formazione compiuto ora atraverso il dolore e la malattia, ora attraverso la spiritualità e la solidarietà.
\end{abstract}

Parole chiave: letteratura di frontiera, letteratura di lingua italiana all'estero, letteratura italiana in Svizzera, autobiografia, racconto di formazione.

\section{Abstract}

The frontier concept is omnipresent in over 250 literary works by Italian émigrés in Switzerland. But with the passage of time and the deline of the genre to a position of secondary importance, the frontier, from a form of discriminating between before and after, of displacing trauma, has been transformed into a metaphor of distance, of preparation either through pain and disease, or through spirituality and solidarity.

Key words: frontier literature, Italian language literature from foreign countries, Italian literature in Switzerland, autobiography, story of formation.

Ciò che caratterizza fondamentalmente la condizione e l'esperienza di vita dei residenti italiani all'estero, e in particolare gli emigrati, è il fatto di aver varcato una frontiera e di risiedere la maggior parte del tempo al di là di quella frontiera. Anzi l'attraversamento di un confine di Stato è proprio quello che distingue l'emigrazione vera e propria dall'emigrazione interna che è stato anch'esso un fenomeno di grande ampiezza nell'Italia del dopoguerra. Vivere all'estero - anche se ai giorni d'oggi la questione della nazionalità si è molto modificata in Europa - significa essere considerato uno straniero de iure nel paese in cui si è emigrati e un cittadino residente all'estero dalla propria nazione. Uno straniero ha sempre avuto meno diritti dei residenti e si è spesso sentito un cittadino di seconda categoria, anche perché nei suoi confronti è quasi sempre scattata la percezione della diversità. Per quanto riguarda la Svizzera, que- 
sta situazione, indipendentemente dallo statuto giuridico, ha subito notevoli variazioni in funzione dei luoghi di emigrazione, della condizione sociale degli emigrati e del periodo in cui è intervenuta l'emigrazione. Culturalmente, ed in particolare linguisticamente, l'integrazione è stata più facile nella Svizzera italiana e nella Svizzera francese che in quella tedesca; è stata accettata senza troppa ostilità nelle attività professionali altamente qualificate e negli ambienti sociali più elevati (anche per il fatto che oggettivamente le differenze di abitudini e di comportamenti erano meno notevoli); ha subito variazioni considerevoli fra la situazione degli anni Sessanta quando l'arrivo massiccio di emigrati fece salire il numero dei residenti stranieri in Svizzera a 800.000, pari al $13,8 \%$ della popolazione, mentre gli italiani erano più di $450.000^{1}$ — scatenando movimenti xenofobi e referendum destinati ad evitare quello che veniva chiamato l' "inforestieramento" - e la situazione degli anni Ottanta e Novanta che ha visto un notevole riflusso degli italiani nel loro paese e una rapida integrazione di coloro che erano rimasti in Svizzera.

Non è perciò sorprendente se fra le 250 opere scritte in italiano e pubblicate in volume da emigrati italiani in Svizzera dalla fine degli anni Cinquanta ad oggi, ${ }^{2}$ la maggior parte di esse rechino la traccia di questa esperienza della frontiera varcata. Ma l'aspetto più interessante di questa presenza non è tanto la frontiera quanto la maniera in cui questa esperienza dell'autore viene «narrativizzata» e diviene elemento costitutivo dell'opera letteraria, che sia un componimento poetico, un racconto o un romanzo: di modo che se per alcuni l'esperienza della frontiera in quanto attraversamento del confine viene riportata nella narrazione come vissuta in un racconto autobiografico o trasposta in quanto vissuto di un protagonista, per altri, per la maggior parte degli autori, la frontiera diviene, in modo molto più metaforico, coinvolgimento dell'identità, riflessione e distanza critica rispetto alla lingua (il dialetto del paese d'origine, l'italiano, la lingua del paese di emigrazione, il dialetto del luogo di residenza, le lingue imparate e / o parlate dai figli), alla cultura (quella arcaica con i suoi valori, quella del nuovo paese, quella della civiltà dei consumi [...]), agli usi e costumi di qua e di là dal confine, alla maniera di vedere la realtà e di viverla nel proprio paese e nel paese di emigrazione.

Va tuttavia precisato che, rispetto ad altri paesi, l'emigrazione in Svizzera ha rappresentato per molti italiani, e perciò per molti autori di questi testi, un trauma più intensamente associato al passaggio della frontiera. Infatti, per

1. Queste cifre, tratte dai dati dell'Ufficio federale della statistica per il 1965 , sono citate in G. MADRASSI, "Riflessi e immagini quotidiane nella narrativa degli emigrati italiani in Svizzera nell'ultimo trentennio", in J.-J. MARCHAND, (a cura di), La letteratura dell'emigrazione. Gli scrittori di lingua italiana nel mondo, Torino: Edizioni della Fondazione Giovanni Agnelli, 1991, p. 39-49.

2. Su questo corpus, cfr. J.-J. MARCHAND, Scrittori e scriventi "emigrati» italiani in Svizzera, in M. GuglielminetTI e J.-J. MARCHAND, Scrittori e scriventi esuli ed emigrati italiani in Svizzera dall'Otto al Novecento, Université de Lausanne, Faculté des lettres, 1996 ("Quaderni italosvizzeri», n. 3), p. 51-116 (bibliografia delle opere a p. 107-16); una bibliografia aggiornata in appendice al saggio: ID., Gli scrittori di lingua italiana residenti in Svizzera, è in corso di stampa in E. Halter (a cura di), Il secolo degli italiani, Zürich: Offizin Verlag, 2003. 
molti anni, gli emigrati stagionali in particolare, venivano fatti scendere dal treno nelle città-frontiera (Chiasso, Briga...), incolonnati e scortati verso una sorta di caserma e sottomessi ad una radiografia e ad una visita medica, per la quale dovevano spogliarsi in parte e presentarsi in quel modo a infermiere e a medici; sul permesso di lavoro degli stagionali veniva inoltre stampigliata una lettera A, che sembrava un marchio infamante. Non va dimenticato che questa scena avveniva solo dieci-vent'anni dopo i tragici eventi della deportazione nazista - eventi spesso presentati in quegli anni in foto e in filmati - e si svolgeva sotto la sorveglianza di vigili per lo più di lingua tedesca; non si dimentichi nemmeno che la J stampigliata sui passaporti degli ebrei che volevano entrare in Svizzera negli anni Quaranta era stato l'atto più infamante della Confederazione negli anni bui della Seconda guerra mondiale. Le finalità puramente igienico-sanitarie di tali operazioni - che per gli stagionali si ripetevano ogni anno - erano certo positive, ma creavano sovente un sentimento di panico in persone impreparate e già angosciate dall'idea di dover affrontare le incognite dell'espatrio e dell'emigrazione. Di solito quella che si potrebbe chiamare la «scena primaria» del trauma della frontiera non viene rappresentata nei testi letterari: appunto perché troppo violenta, perché troppo poco sopportabile per essere rappresentata direttamente dagli autori che l'hanno vissuta. Lo stesso Leonardo Zanier, che, fin dagli anni Sessanta, ha affrontato più spesso e con maggiore riflessione teorica, come vedremo, il tema della frontiera in tutte le sue implicazioni concrete, non ha mai evocato questa scena direttamente prima del 2002, e lo ha fatto, per ora, non in una opera letteraria, ma in una relazione di convegno:

Sul mio impatto con la Svizzera, 1956, non ho ancora scritto niente. Quanto segue è quindi assolutamente inedito [...] Accenno solo alla visita a Chiasso, la polizia che fa scendere dal treno tutti i permessi A (lavoratori stagionali). Spesso anche i B (annuali). La lettera A è stampigliata con un grosso timbro blu sul contratto [...] Prima associazione da brivido. Da allora sono passati molti anni. Le J stampigliate su altri passaporti [...]

Le successive fasi sono note: in fila in mutande, con il passaporto in mano, radiografia ai polmoni, ispezione delle gengive e dei denti, strizzatina alle palle, ecc. Organi che, a vent'anni, sono perlopiù in buono stato. ${ }^{3}$

Solo due autori, che non hanno vissuto personalmente l'esperienza, narrano la scena: uno, Saro Marretta, venuto in Svizzera come insegnante, la evoca in modo piuttosto ironico in un racconto in terza persona, Il paese finiva alla stazione, scritto nel 1977:

A Chiasso ci pensarono però i colleghi d'oltre frontiera a farli scendere. Avevano visto Ombra con gli occhi sgranati di paura e s'erano precipitati a bloccargli

3. L. ZANIER, Dal friulano all'italiano: rischiando di morire [...], in J.-J. MARCHAND (a cura di), Letteratura di lingua italiana in Svizzera. Scrittura e lingua, Université de Lausanne, Faculté des lettres, 2003 («Quaderni italo-svizzeri», n. 5) (in corso di stampa). 
le mani. Li portarono tutti in un casermone bianco, disinfettato e li misero in fila per la visita medica. Perché lo stagionale quando entra dev'essere sano. Quando esce nessuno lo visita e sono affari suoi se s'è ammalato. Anche Cantante cominciò a non sentirsi più sicuro con gli occhi delle infermiere quarantenni addosso e degli uomini col camice bianco che se non erano medici o infermieri, ai suoi occhi potevano essere degli sbirri travestiti. ${ }^{4}$

L'altro autore è Attilia Fiorenza Venturini, venuta a vivere a Lugano dopo il matrimonio con un ingegnere svizzero: ciò che spiega perché non abbia esitato a dare al proprio romanzo-saggio il titolo considerato tabù di Nudi col passaporto. La verità sull'emigrazione italiana in Svizzera. ${ }^{5}$

Altri autori invece si contentano di alludere solo indirettamente alla scena, per studiare più ampiamente la problematica legata a tutte le fasi della vicenda migratoria. In questi testi inoltre il taglio saggistico o polemico prende il sopravvento, come per evitare qualsiasi fissazione affettiva sulla scena, che probabilmente nemmeno loro hanno vissuta di persona. Si tratta di Diario di un emigrante di Marco Patruno del $1975^{6}$ e di Passaporti prego di Rosanna Ambrosi del 1985,7 che pur narrando scene di emigrazione, con taglio saggistico, segnalano vie alternative, danno informazioni per rendere meno duro l'impatto dell'emigrazione.

Tuttavia le opere di coloro che hanno effettivamente affrontato da immigrati questa prova della frontiera hanno spesso per argomento il periodo anteriore o quello posteriore all'attraversamento del confine. Per molti racconti, la frontiera, senza essere esplicitamente evocata, fa da discrimine fra un prima e un dopo rispetto alla fase migratoria, e delimita nello stesso tempo due campi tematici. Il "prima", riferito al mondo delle origini, dell'infanzia, della gioventù, è lontano nel tempo e nello spazio. È il mondo idealizzato e mitizzato della terra dei padri, la culla protetta dalla dolcezza materna, il paese delle radici: il luogo del calore, della luce e del sole, dello sfavillio del mare, e della purezza della campagna o della montagna. Chi la rappresenta nel modo più assoluto e più tipico, fin dal titolo della raccolta, è Toto Mazzara in Amata terra mia. ${ }^{8}$ $\mathrm{Ma}$ in altri autori, questo luogo perde anche quei connotati un po' patetici e un po' nostalgici che costituiscono un «cliché» un po' troppo riduttivo della letteratura di emigrazione. Talvolta, l'uso del dialetto dà a questa tematica una dimensione più genuina, soprattutto se l'autore mira a più alti valori o a più forti simboli dietro alle immagini più frequenti dell'infanzia, come nell'evocazione di questa fontana di famiglia di Franco Aste, che nel componimento La nosa fontana fa comparire dietro all'oggetto descritto tutta una simbologia di forza (il granito), di vita (l'acqua) e di santità (l'acquasantiera): «[...] Ma adès / che lù / da 'm pèz l'è 'm paradis / e noi / sém chi zo bas / su 'n de sta tèra / podém

4. S. Marretta, Il paese finiva alla stazione, Berna: Benteli, 1977, p. 42.

5. Con prefazione di M. Frisch, Milano: Pan.

6. Milano: Cammino.

7. Zurigo: Federazione delle Colonie Libere.

8. Milano: Club degli Autori, 1977. 
amiràr / el so capolavoro: / vardém 'ncantài / colàr l'acqua come la vita / da sto préôm / che 'l nòno de nòs nono / l'à trasformà en na fontana / che per noi / la è anca n'acquasantel / el pù bel e 'l pù prezios / del mondo». ${ }^{9}$ E nello stesso senso, ma con una ricerca di simbologie più pagane e con un effetto più corale, va la descrizione che Zanier dà, in lingua friulana, della festa delle «cidulas», quelle rotelle di legno infuocate che i giovani lanciano nelle notti d'estate facendo un voto: «mi recuardi las cidulas / tratas da un pruc / in ta not // la lô or coda di fôc / s'incorsin sot las stelas // [...] // mi recuardi la vôs / dai zovins sul pruc / 'in onor [...] in favôr' // contin i nons las speranças / i amôrs di un païs // [...]». ${ }^{10}$ Tuttavia il mondo delle origini, prima dell'attraversamento della frontiera è spesso anche quello che spinge all'emigrazione: è un mondo fatto di durezza, di povertà, di disoccupazione, quello della patriamatrigna (e non più della terra-madre) che costringe i figli a varcare i confini e a raggiungere la schiera di quelli che Zanier designa con la perifrasi ironica di coloro che sono Liberi [...] di dover emigrare (Libers [...] di scugnî lâ). ${ }^{11}$

Dall'altra parte della frontiera varcata, inizia il «dopo», cioè l'ampia tematica del mondo nuovo: del paese e della vita dell'emigrazione, da cui si tornerà magari, ma già estranei, e sempre di più di anno in anno, al proprio paese. Il momento cronologicamente più alto in questa evocazione della nuova vita, si situa poco dopo il passaggio da quel luogo «indicibile» in cui l'emigrato si è presentato «nudo col passaporto». Due racconti fra i più importanti del primo ventennio dell'emigrazione italiana in Svizzera cominciano significativamente alla stazione di Zurigo: in Io sono un «cinq" di Giampiero Montana, ${ }^{12}$ che narra il primo anno nella Svizzera tedesca di un giovane emigrato e in Stagionali e rami secchi di Attilia Fiorenza Venturini, ${ }^{13}$ un romanzo storico che racconta le vicende di un gruppo di emigrati all'epoca dei violenti moti antiitaliani avvenuti a Zurigo nel 1896. Infatti narrativamente la vera frontiera fra passato e futuro non si situa al confine - nonostante quel rito di passaggio sconcertante -, ma al momento in cui l'emigrato, per lo più solo, deve affrontare la sua nuova vita. Come dimostra il racconto di Saro Marretta Il paese finiva alla stazione, ${ }^{14}$ il treno, con le sue carrozze partite dall'Italia, con le persone che hanno fatto tutto il viaggio o gran parte di esso insieme, ricostruendo una

9. In Frammenti di vita. Presentazione di J.-J. Marchand, Calliano: Manfrini, 1991.

10. «mi ricordo las cidulas / lanciate da un colle / la notte // le loro code di fuoco / s'intrecciano sotto le stelle // [...] // mi ricordo le voci / dei giovani / dei giovani sul colle / 'in onore [...] in favore' // raccontano i nomi le speranze / gli amori di un paese // [...]» (Traduzione dell'autore). In onor / in favor, in Che Diaz [...] us al meriti, Ajello del Friuli: Circolo Culturale A. Colavini, 1976 (ora in Den Wasserspiegel schneiden / Sot il pêl da l'àga, Zurigo: Limmat Verlag, 2002).

11. Milano: Garzanti, 1977.

12. Io sono un cinq. Un emigrato italiano in Svizzera, Firenze-Empoli: Poligrafo Toscano, 1961. Il soprannome spregiativo "cinq" o "cingali», che si riferiva al numero "cinque» ricorrente nel gioco della morra, veniva dato ai primi emigrati italiani, in particolare nella Svizzera tedesca.

13. Milano: Pan, 1976. I «rami secchi» sono coloro che non riescono ad adattarsi alla vita dell'emigrazione e che sono costretti a ritornare nel loro paese.

14. Cfr. nota 3. 
microsocietà legata ancora alla fase anteriore al passaggio della frontiera, è in qualche modo un' «estensione» del paese che si lascia. La stazione di destinazione diviene di fatto l'effettiva frontiera, il discrimine tra il passato e il futuro, tra la vita in patria e la vita da emigrato o almeno da straniero, il momento in cui il protagonista prende coscienza di essere giunto in un paese diverso dal suo e di dovere affrontare una realtà nuova.

Altri narratori, forse ancora più numerosi, eludono totalmente questa componente del viaggio iniziale, con i suoi traumi della frontiera varcata, per trasporla in una realtà diversa, sempre concreta ma posta in un altro contesto. Stefano d'Acquisto in Una stella in un bicchiere di whisky ${ }^{15}$ usa quale motivo centrale del suo racconto l'iniziazione che devono superare i giovani marinai quando attraversano la linea dell'equatore: il disagio anche fisico, il timore, il disgusto spinto fino alla nausea dalla dura prova dell'attraversamento della frontiera vengono trasposti nella descrizione della disgustosa prova dell'assaggio del pesce marcio imposta a chi varca la frontiera dell'equatore. D'altra parte, nel racconto la scena simbolica e iniziatica viene subito seguita da un episodio in cui in una subitanea tempesta il marinaio deve affrontare i reali pericoli della navigazione e dimostrare con i fatti che la sua trasformazione in vero e proprio marinaio è stata compiuta. Da allora, la vita del protagonista verrà sempre segnata dalla "pendolarità", dall'attraversamento continuo di frontiere: tra i mari del mondo solcati sulle navi e la casa materna in Sicilia, fra il desiderio di capire la propria natura e la tentazione dell'evasione nell'alcool, tra il bisogno di scrittura e l'esigenza del delirio etilico per ricordare o per raggiungere una presunta lucidità. Ma la presa di coscienza dell'alterità, e della problematica dell'attraversamento della frontiera viene anche rappresentato con un allontanamento cronologico rispetto alla scena dell'espatrio dell'emigrato: Luisa Moraschinelli, per esempio, che in altri racconti affronterà la tematica, in gran parte autobiografica, della vita di emigrata, rappresenta nel racconto Ricordi di guerra. Una ragazza valtellinese racconta, ${ }^{16}$ l'intuizione da parte di una giovane contadina valtellinese del dramma dello sradicamento nella presenza di un gruppo di ebrei fuggiti da Zagabria dopo l'armistizio dell'8 settembre 1943 e la conseguente occupazione tedesca dell'Italia. La frontiera interna, anche sociale, economica e culturale, che hanno varcato questi sfollati ebrei, questi cittadini rifugiati in montagna, diventa per la protagonista oggetto di riflessione su un certo numero di idee preconcette del suo paese e della sua infanzia. ${ }^{17} \mathrm{Ed}$ anche in questo caso, questo attraversamento apparentemente simbolico della frontiera viene confermato subito dopo da descrizioni di tragici attraversamenti, tentati e spesso drammaticamente falliti, tra Valtellina e Svizzera compiuti dagli stessi ebrei perseguitati dai nazisti.

15. Milano: Joppolo, 1994.

16. Sondrio: Bonazzi, 1995.

17. Il libro comporta difatti la dedica seguente: «Dedico questo libro agli "Zagabri" confinati all'Aprica durante la guerra per la buona impressione che hanno suscitato nel mio animo di bambina». Sullo stesso tema verte il romanzo di Attilia Fiorenza VENTURINI, La frontiera del sole. Ebrei veneziani verso la salvezza in Ticino, Lugano: Editions transalpines, 1994. 
Sia per ragioni di distanza psicologica o narrativa, sia per evoluzione naturale delle opere scritte da emigrati italiani in Svizzera, ${ }^{18}$ la produzione letteraria passa assai rapidamente da una tematica incentrata sulle problematiche dell'emigrazione (in scritti sia di tipo autobiografico, sia di tipo più liberamente narrativo) ad opere svincolate da riferimenti espliciti al motivo migratorio. In alcuni testi la presenza della frontiera rimane forte ma si manifesta già in modo metaforico. Le metaforizzazioni più frequenti sono quelle della malattia e della guerra che portano il protagonista sulla "frontiera» della morte. In Diario di un viaggio nel coma e dintorni, pubblicato nel 1991, Francesco De Giovanni ${ }^{19}$ narra l'esperienza di chi raggiunge il confine vicino alla morte e, sfioratolo, ritorna nel mondo dei vivi: è un percorso che ha qualcosa di affascinante e di raccapricciante appunto perché, contrariamente a quello varcato dagli emigrati, questo è un confine che, se fosse valicato, sarebbe senza ritorno. Ma è anche una narrazione che potrebbe avere qualcosa in comune con l'avventura dell'emigrazione, poiché, anche se le condizioni degli emigrati europei del dopoguerra sono state diverse da quelli che varcavano l'oceano nel primo Novecento, il passaggio della frontiera ha continuato ad essere segretamente associata al timore del non ritorno, o addirittura a un presentimento di morte. ${ }^{20} \mathrm{Ma}$ la stessa tematica può portare ad una rappresentazione del tutto diversa. Storia di vita vissuta di Alessandro Costacurta ${ }^{21}$ narra il calvario di chi, da un'operazione neurologica all'altra, ha rischiato varie volte di perdere la vita: tuttavia non è quel limite invalicabile varie volte sfiorato che costituisce il motivo centrale del racconto, poiché nella forte fede cristiana dell'autore tale evento rappresenterebbe il congiungimento con Dio, bensì le costanti ed assillanti sofferenze provate sull'orlo stesso della morte, che potrebbero far crollare il protagonista, se non ci fosse in lui la volontà di offrirle a Dio come riscatto del male nel mondo; la frontiera rifiutata non è perciò più la morte, ma l'attimo di cedimento alla sfida del dolore.

In altri racconti, anche se la frontiera evocata sembra più vicina ad una rappresentazione materiale, ci si accorge che quella che è determinante nel racconto o nel componimento è la frontiera metaforica. Ne I bagni di Caldiero del 1991,22 Rosanna Ambrosi ambienta il racconto in un viaggio di ritorno in Italia causato dalla morte del padre: è l'occasione di un lungo ripensamento sulla propria vita, punteggiato da vari ricordi, segnalati nel testo dall'uso del corsivo: a questo viaggio fa da contraltare un altro viaggio che la protagonista fece in senso inverso per andare a vivere con il marito, dopo la lunga bat-

18. Su questo punto rinviamo al nostro saggio «La letteratura dell'immigrazione italiana in Svizzera", in AA.VV., Lingua e letteratura italiana nel mondo oggi. Atti del XIII Congresso dell'Associazione Internazionale per gli Studi di Lingua e Letteratura Italiana. Perugia, 30 maggio-3 giugno 1988, Firenze: Olschki, 1991, II, p. 457-79.

19. Münchenbuchsee: Arcobaleno.

20. La similitudine era frequente nei testi dell'emigrazione transoceanica «emigrare è un po' morire».

21. Susegana: Arti grafiche Conegliano, 1987 (con il sottotitolo: Piccolo riassunto).

22. Bellinzona: Casagrande. 
taglia che l'aveva opposta alla famiglia e nella città natia. Ma la vera frontiera che viene affrontata nel racconto è quella del tempo: rispetto ai racconti di emigrazione tradizionali, non c'è più solo un prima o un dopo rispetto al passaggio della frontiera, ma c'è ancora un "terzo tempo», di cui occorre prendere coscienza varcando tutta l'esperienza di un ventennio vissuto all'estero (la nascita dei figli, la separazione dal marito, l'incontro con un altro compagno, la morte dei genitori, il difficile dialogo con i figli) [...] In Animaterra di Gemma Capone del $1998^{23}$ è la frontiera varcata da altri, dai figli — uno partito in America e l'altra ricoverata in un istituto per handicappati - a costringere la protagonista a riconquistare uno spazio proprio: ora facendo il punto sulla propria vita, ora tornando mentalmente alla propria infanzia, ora proiettandosi nel futuro con la volontà di completare la propria cultura. Il racconto si costruisce, anche qui tipograficamente in un'alternanza di tondo e di corsivo, fra la comparsa di questa doppia frontiera creata dalla partenza dei figli e la conquista progressiva — anche attraverso la scrittura — di uno spazio vitale e psicologico alternativo.

A queste conquiste individuali di territori interni, che tendono a contestare o a respingere frontiere psicologiche, fa da contrasto l'ampia apertura sociologica e politica di Leonardo Zanier sul concetto di frontiera. Mentre gli scritti che abbiamo analizzato precedentemente riportavano all'esperienza individuale la «scena primaria» del valico della frontiera dell'emigrato, Zanier l'allarga ad un'ampia considerazione storica, mettendo in evidenza l'assurdità delle guerre, in particolare quelle combattute sul fronte carnico durante la prima e la seconda guerra mondiale; due intere raccolte sono dedicate al tema: Che Diaz [...] us al meriti2 e l'antologia Cjermins / Grenzsteine / Mejniki / Confi$n i .^{25}$ Più generalmente ancora, la riflessione si allarga a tutti i fanatismi che hanno indotto gli uomini a tracciare frontiere: politiche certo, ma anche sociali e religiose fin dai tempi della Riforma e della Controriforma, tanto che nella sezione sugli intolleranti intitolata Gott mit uns si trovano accomunati san Carlo Borromeo e il riformatore zurighese Zwingli.

Se prendiamo in considerazione le opere che si allontanano ancora maggiormente dalla "materialità» della frontiera, possiamo costatare che la presenza del confine si manifesta in molte opere di emigrati relative alle relazioni umane, e in particolare all'amicizia e all'amore. Si tratta per lo più di una messa in scena positiva della "frontiera», poiché sono appunto queste relazioni umane fatte di fraternità e di sentimenti amorosi, che permettono di superare le frontiere che - per ragioni di superstizioni, di credenze, di assurde tradizioni tenderebbero ad ostacolare l'apertura al prossimo. Nella mente dell'emigrato viene, per esempio, spontaneo rappresentare l'incontro con la donna amata come il punto di arrivo di un lungo viaggio in cui ogni protagonista ha attraversato paesi e varcato frontiere prima del felice congiungimento. Enzo Liot- 
ta usa addirittura la metafora del polline che, portato dal vento, attraversa i continenti, in Come polline al vento, per poi iniziare con queste parole il componimento d'amore eponimo della raccolta:

Lo scirocco aiutava il treno

a salire verso il Nord

nel continente.

Quanta terra, quanti paesi

fermenti di vita che scartai, per arrivare dove?

A quella sveva fermata

dov'eri tu che aspettavi senza conoscermi, $[\ldots]^{26}$

Per altri la frontiera non è più quella che si varca personalmente, anche se metaforicamente, ma quella che la presenza di persone di nazionalità e di culture diverse porta in mezzo a noi nel nostro quotidiano. L'emigrato dopo essere stato rappresentato come oggetto di estraneità, e averne sentito le umiliazioni, le offese o almeno i disagi, diviene l'iniziatore di un rapporto di fraternità, di superamento delle frontiere, della apparente diversità di nazione, di pelle, di lingua e di cultura. In alcuni casi è proprio il modello evangelico a costituire il motivo ispiratore della scena di condivisione dell'amore fraterno e di riscatto morale, come in Gesti di amore nella raccolta Fame d'amore di Franco Aste:

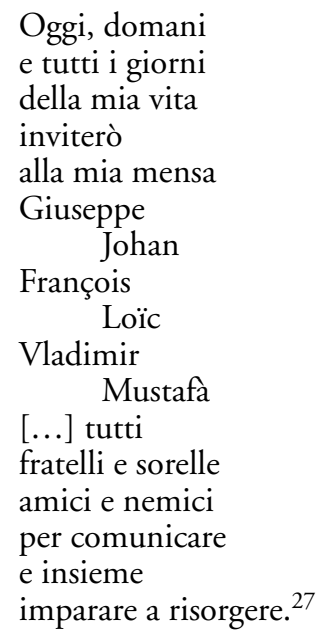

Ma la frontiera nella sua funzione simbolica può anche rappresentare una protezione desiderata, una difesa della sfera privata, una separazione tra l'interiorità con il suo mondo costruito e l'esteriorità concepita come uno spazio 
sterminato che potrebbe privarci della nostra identità. La porta, per esempio, nella sezione poetica L'appartamento del 1984 di Alida Airaghi, si presenta come una vera e propria frontiera protettiva tra la fragilità interna e l'ostilità esterna:
Chi entra non la guarda nemmeno preso com'è a ripassarsi la futura scena.
Lei non prepara a niente, non assomiglia
a chi nasconde; è come tante, appena scura, tarda ad aprirsi.
Ma chi evade ne osserva col peso la resistenza.
Sente che le premono addosso i folletti
domestici, gli oggetti prigionieri.
Senza la porta, la casa sarebbe
già scappata da se stessa, sparsa
nelle strade, dietro il visitatore incauto.
È un bene che sia così pronta a richiudersi,
fedele come una serva, in silenzio come una morta. ${ }^{28}$

Si noterà che, sebbene la porta-frontiera sia connotata positivamente, essa è già subdolamente associata ad una morta. Ma con l'andare del tempo, questa chiusura dell'io all'interno di una frontiera protettiva andrà accrescendosi nell'opera dell'Airaghi, fino a giungere ad un doppia chiusura pressoché ermetica in seguito ad una sorta di esilio volontario a Zurigo con il marito (il poeta Siro Angeli), ed alla successiva morte di lui. La raccolta Il lago del 1996 rende conto di una situazione di profonda estraneità, di una sorta di pietrificazione del mondo circostante e di inquietante raggelamento della natura: la città di Zurigo a cui allude sembra essersi vuotata da ogni vita ed essere caduta in una forma di ibernazione, come se fosse calato un sipario di gelo tra lei e il mondo circostante.

Con l'assumere la funzione di una metafora più strettamente personale, legata all'individualità del poeta, a fungere da equivalente di una raffinata espressione di incomunicabilità, la frontiera rischierebbe di chiudersi nell'espressione di una non comunicazione un po' intellettualistica.

E difatti Silvana Lattmann, che nelle raccolte Fessura del $1983^{29}$ e Il viaggio del $1987,{ }^{30}$ ha mostrato come, senza la minima allusione alla frontiera, si possano comporre opere in cui un "oggetto" sia presente nonostante la sua assenza, non manca di rilevare che il rischio di una chiusura nelle proprie "frontiere» costituisce una minaccia incombente su ognuno di noi:

\footnotetext{
Di fronte a questa tua risposta

potrei dirti

che abitiamo ognuno il suo cassetto

in un armadio cosmico. ${ }^{31}$
}

28. In W. SiTI (a cura di), Nuovi poeti italiani, Torino: Einaudi, 1984.

29. Bellinzona: Casagrande.

30. Bellinzona: Casagrande.

31. Fessura, cit. 